\title{
ARTICLE
}

\section{Gekko japonicus genome reveals evolution of adhesive toe pads and tail regeneration}

Yan Liu ${ }^{1, \star}$, Qian Zhou ${ }^{2, \star}$, Yongjun Wang ${ }^{1, \star}$, Longhai Luo ${ }^{2, \star}$, Jian Yang ${ }^{1, \star}$, Linfeng Yang ${ }^{2, \star}$, Mei Liu 1 , Yingrui Li ${ }^{2}$,

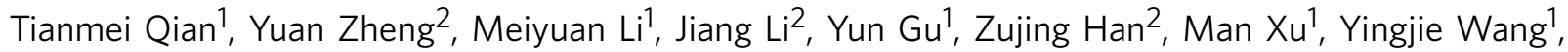
Changlai Zhu', Bin Yu ${ }^{1}$ Yumin Yang ${ }^{1}$, Fei Ding ${ }^{1}$, Jianping Jiang ${ }^{3}$, Huanming Yang ${ }^{2,4} \&$ Xiaosong Gu ${ }^{1}$

Reptiles are the most morphologically and physiologically diverse tetrapods, and have undergone 300 million years of adaptive evolution. Within the reptilian tetrapods, geckos possess several interesting features, including the ability to regenerate autotomized tails and to climb on smooth surfaces. Here we sequence the genome of Gekko japonicus (Schlegel's Japanese Gecko) and investigate genetic elements related to its physiology. We obtain a draft G. japonicus genome sequence of $2.55 \mathrm{~Gb}$ and annotated 22,487 genes. Comparative genomic analysis reveals specific gene family expansions or reductions that are associated with the formation of adhesive setae, nocturnal vision and tail regeneration, as well as the diversification of olfactory sensation. The obtained genomic data provide robust genetic evidence of adaptive evolution in reptiles.

\footnotetext{
${ }^{1}$ JS Key Laboratory of Neuroregeneration, Co-innovation Center of Neuroregeneration, Nantong University, Nantong 226001, China. ${ }^{2}$ Beijing Genomics Institute, BGI-Shenzhen, Shenzhen 518083, China. ${ }^{3}$ Chengdu institute of biology, Chinese Academy of Sciences, Chengdu 610041, China. ${ }^{4}$ James D. Watson Institute of Genome Sciences, Hangzhou 610041, China. * These authors contributed equally to the work. Correspondence and requests for materials should be addressed to H.Y.

(email: yanghm@genomics.cn) or to X.G. (email: nervegu@ntu.edu.cn).
} 
R eptiles diverged from early tetrapods in the late Carboniferous period approximately 310-320 million years ago $(\text { Myr ago })^{1}$. Since then, these ancient amniotes have spread across the world and adopted diverse morphologies and habitats (aquatic and terrestrial) $^{2}$. More than 10,000 reptile species have been documented and they are classified into the following four orders: Crocodilia, Testudines, Squamata and Sphenodontia ${ }^{3}$. Squamate reptiles represent the most diverse radiation of terrestrial vertebrates and are traditionally split into two major clades, Iguania and Scleroglossa ${ }^{3,4}$. The latter clade includes the terrestrial Gekkonidae, which consists of $\sim 1,450$ species in 118 genera and comprises $25 \%$ of all described lizard species ${ }^{5}$. Geckos have evolved in a terrestrial niche, where selective pressure ${ }^{6}$ has resulted in traits such as small body size, agility and nocturnal habits. Most gecko species possess adhesive toe pads, which enable them to capture live food more easily, and flee from their predators by scaling vertical or even inverted surfaces ${ }^{7}$. This ability is due to the presence of setae, microscopic hair-like outgrowths of the superficial layer of the subdigital epidermis, which comprise the primary components of the adhesive apparatus ${ }^{7,8}$. The most interesting and physiologically significant trait in geckos is their ability to voluntarily shed or autotomize their tails to escape from attack, they then regenerate a new tail ${ }^{9}$. Given these interesting characteristics, geckos have been used in studies on regenerative processes, and their adhesive mechanism has been examined for the development of bioinspired technologies ${ }^{10}$. The availability of genome sequence data would significantly contribute to deciphering the evolutionary events related to lineage-specific anatomical adaptations.

To date, the genomes of several reptilian species, including species from Squamata reptiles (Anolis carolinensis, Python molurus bivittatus and Ophiophagus hannah), Crocodylia reptiles (Alligator sinensis, Alligator mississippiensis, Gavialis gangeticus and Crocodylus porosus) and Testudines reptiles (Chelonia mydas, Pelodiscus sinensis and Chrysemys picta bellii) have been successfully sequenced ${ }^{11-15}$. These results have filled the genomic gap between amphibians and birds by providing genomic information on a wide variety of morphologically and physiologically distinct species. However, genomic data from the species comprising the family Gekkonidae, which is an important clade of Lepidosauria and one of the earliest branches off the squamata phylogenetic tree, have not yet been acquired. In this study, the genome of G. japonicus (Schlegel's Japanese Gecko) is sequenced and annotated, which provides valuable insights into the adaptive evolution of geckos as well as the genomic basis of their characteristic traits. For example, our data reveals that the expansion of $\beta$-keratin gene family is essential to the clinging ability of G. japonicus, and the evolution of the opsin gene is correlated with its visual adaptation. Moreover, some positive selected genes (PSGs) potentially involved in the tail regeneration are identified as well. In addition, developing a genomic resource associated with geckos is helpful in understanding the evolutionary history of Lepidosauria.

\section{Results}

Sequencing and annotation of the G. japonicus genome. The genome of an adult male G. japonicus was sequenced and assembled (Supplementary Figs 1-3 and Tables 1-3). The draft genome sequence of $G$. japonicus was $2.55 \mathrm{~Gb}$ in size, $\sim 50 \%$ larger than that of Anolis carolinensis, a lizard that belongs to the Iguania of Squamata, making it the largest sequenced genome to date among all reptiles with available genome data ${ }^{11-14}$. The assembly quality was assessed using 10 fosmid clones and RNA-Seq data (Supplementary Fig. 4 and Tables 4-6).

Repeated elements were annotated using Repeat Masker program $^{16}$. The repeats comprised $48.94 \%$ of the genome, and

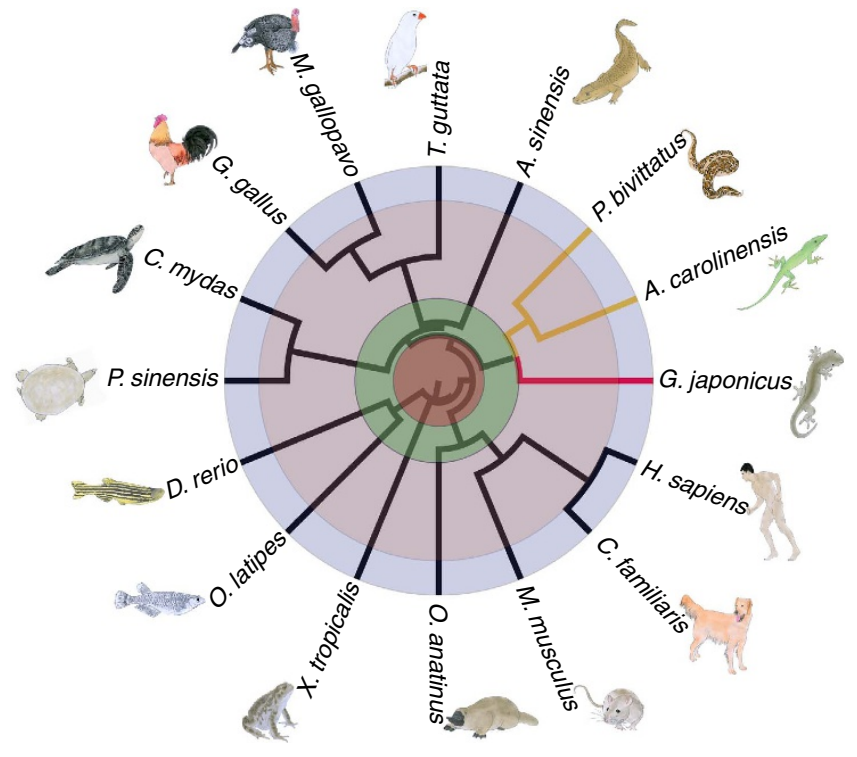

Figure 1 | Phylogenetic analysis of the whole-genomes of 6 reptilian species and $\mathbf{1 0}$ additional vertebrate species. The species in the phylogenetic tree include Danio rerio (D. rerio), Xenopus tropicalis ( $X$. tropicalis), Chelonia mydas (C. mydas), Pelodiscus sinensis ( $P$. sinensis), Alligator sinensis ( $A$. sinensis), Python molurus bivittatus ( $P$. bivittatus), Anolis carolinensis (A. carolinensis), Gekko japonicus (G. japonicus), Taeniopygia guttata (T. guttata), Gallus gallus (G. gallus), Ornithorhynchus anatinus (O. anatinus), Canis familiaris (C. familiaris), Mus musculus (M. musculus), Homo sapiens (H. sapiens), Oryzias latipes (O. latipes) and Meleagris gallopavo (M. gallopavo). Before the Permian period is represented in brown. The Permian period to the Triassic period is represented in green. The Triassic period to the Paleogene period is represented in purple. The Paleogene period to the present is represented in blue.

most were transposable elements, making up $48.02 \%$ of the assembly (Supplementary Fig. 5 and Tables 7 and 8). The GC content in G. japonicus genome was about $45.5 \%$, which is slightly higher than in genome of other amniotes (for example, Anolis. carolinensis, 40.3\%; Gallus gallus, 41.5\%; Homo sapiens, $40.8 \%)$. The GC content was primarily distributed in introns, the intergenic regions and CDS regions (Supplementary Figs 6 and 7). Collectively, the above data indicate that the large genome size of $G$. japonicus may primarily result from the greater abundance of repeated sequences compared with other genomes, such as that of $A$. carolinensis (Supplementary Tables 9-12).

A total of 22,487 coding regions and 1,302 non-coding RNAs were predicted in the G. japonicus genome (Supplementary Tables 13-15), and $\sim 95.08 \%$ of the coding regions were functionally annotated (Supplementary Table 16). Then, the orthologous and paralogous genes were clustered and compared among different species background (Supplementary Figs 8-10 and Tables 17 and 18). The data revealed that G. japonicus had 11,513 orthologous gene pairs compared with A. carolinensis, and the mean identity reached up to $72.37 \%$ (Supplementary Fig. 9 and Table 18). A comparison of gene data among four reptiles (G. japonicus, An. carolinensis, Al. sinensis and C. mydas) revealed $\sim 13,478$ orthologous gene families in total, of which 7,546 were shared by four species, 1,240 were specific to G. japonicus, 798 were specific to An. carolinensis, 911 were specific to Al. sinensis and 673 were specific to C. mydas (Supplementary Fig. 10). These species-specific unique orthologous may be involved in lineage-specific adaptations.

Evolutionary analysis of the $G$. japonicus genome. We assessed evolutionary relationships among morphologically and 
a

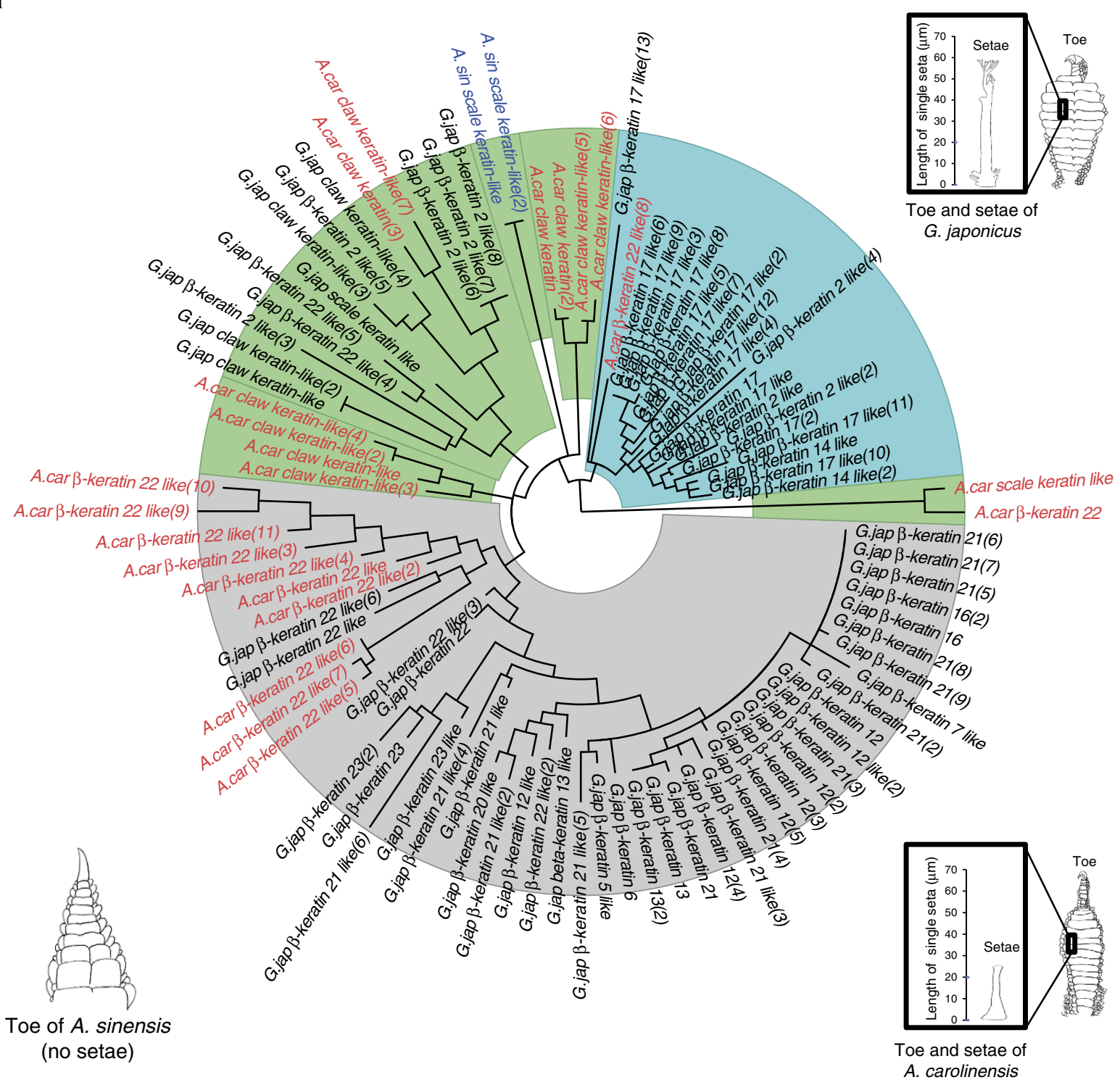

b

GL343369 of $A$. carolinensis

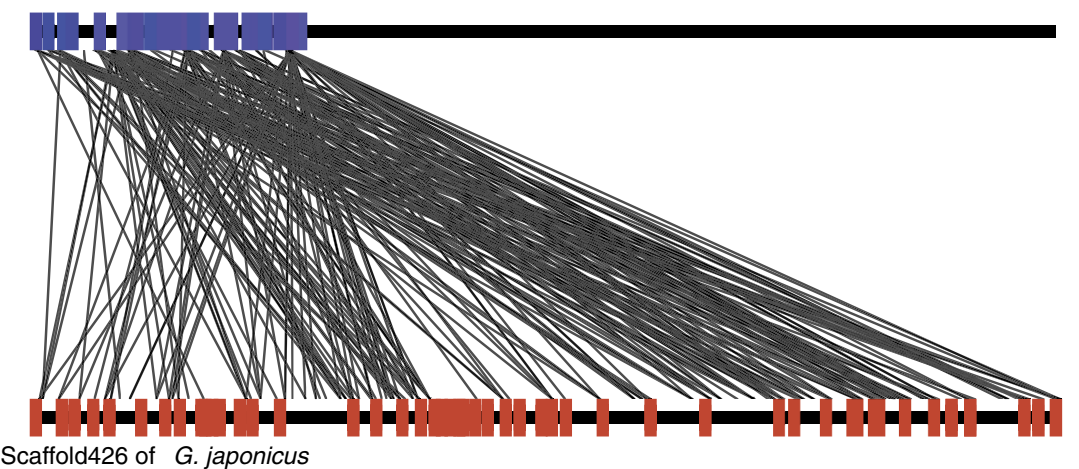

Figure 2 | Phylogenetic tree of $\boldsymbol{\beta}$-keratin families from G. japonicus, An. carolinensis and Al. sinensis. (a) The $\beta$-keratins in black font belong to G. japonicus, those in red belong to An. carolinensis and those in blue belong to Al. sinensis. Gene copy number is listed in parentheses. The green background denotes $\beta$-keratins in scales and claws. The grey background denotes $\beta$-keratins in setae. The blue background denotes $\beta$-keratins in digital scales and pad lamella for supporting setae. A schematic diagram of toe of G. japonicus, An. carolinensis and Al. sinensis, which possess branched setae, unbranched setae and no setae are presented, respectively. The setae of gecko G. japonicus are $\sim 60 \mu \mathrm{m}$ in length, and that in An. carolinensis is $\sim 25 \mu \mathrm{m}$. (b) Synteny diagram of $\beta$-keratin genes in A. carolinensis (upper line: GL343369, blue: $23 \beta$-keratin genes) and G. japonicus (lower line: scaffold 426, red: $48 \beta$-keratins). 
ecologically diverse reptiles by constructing a phylogenetic tree using the whole-genomes of 6 reptilian species and 10 other vertebrates. The results support the view that the species of Gekkota diverged early from the group containing Anolis and Python $200 \mathrm{Myr}$ ago ${ }^{17}$, when Gondwanaland separated from Laurasia $^{18}$. This time period is earlier than previous reported ${ }^{19}$, but later than the divergence of Sphenodon ${ }^{20}$. A. carolinensis clusters with $P$. bivittatus rather than with $G$. japonicus, indicating that $A$. carolinensis and $P$. bivittatus have a much closer genetic relationship, even though $P$. bivittatus and G. japonicus have traditionally been classified as scleroglossans ${ }^{4}$. The phylogenetic tree shows that the crocodilian lineage diverged from chelonian about $250 \mathrm{Myr}$ ago and clusters in the same clade with birds (Fig. 1). A second phylogenetic tree constructed with conserved housekeeping proteins shows consistent results with these findings (Supplementary Fig. 11). The cladogram of whole-genomes clearly shows the evolutionary relationships among these selected amniotes, and the whole genome resource will reduce the ambiguities of phylogeny data when assessed by previous methods based on morphological characteristics or few genes ${ }^{17,19}$.

Adaptive evolution of setae $\beta$-keratins in G. japonicus. The emergence of novel lineage-specific morphological features is always accompanied with genes duplications and diversions. A prominent example is the large-scale duplication of $\beta$-keratin genes that has been crucial to the evolution of scales, claws, beaks and feathers in reptiles and birds. The emergence of setae in geckos also resulted from the duplication and diversion of $\beta$-keratin genes. The clinging ability of geckos depends primarily on spade-like adhesive setae ${ }^{21}$. Previous reports have indicated that setae are comprised of a corneous material largely made of $\beta$-keratins of $8-22 \mathrm{kDa}$, with cysteine-rich proteins located in setae/spatula, and glycine-rich proteins in the $\beta$-layer of epidermis ${ }^{22}$. In addition, $\beta$-keratins have high isoelectric points with positive charges that enhance Van der Waals adhesion in the setae. These physicochemical properties indicate that $\beta$-keratin proteins are key to the clinging ability of setae ${ }^{23}$. To investigate whether the $\beta$-keratin genes are associated with varying adhesive ability in different reptile species, we retrieved the $\beta$-keratin genes from the genomes of G. japonicus, An. carolinensis and Al. sinensis, which possess branched setae, unbranched setae and no setae, respectively. Our analysis showed that the $\beta$-keratin gene families have undergone major expansion corresponding with adhesive ability (Supplementary Table 19). G. japonicus, An. carolinensis and Al. sinensis contain 71, 23 and $2 \beta$-keratin genes, respectively (Fig. 2, Supplementary Table 20). The majority of the extensively expanded families in G. japonicus genome contained the setae $\beta$-keratins genes important for setae production. These $\beta$-keratin proteins are usually characterized with S-core box (SEVTIQPPPCTVVVPGPVLA), cysteine-rich and low-molecular weight $(\sim 10 \mathrm{kDa})$, which had been investigated in Gekko gecko. In the G. japonicus genome, 35 setae $\beta$-keratins with featured S-core box were identified among all $71 \beta$-keratins by aligning them with amino acid sequences of S-core box (sequence similarity $\geq 70 \%$ ). Additional important features associated with the above $\beta$-keratins, such as cysteine content and molecular weight were summarized in Fig. 3a and Supplementary Table 20. Furthermore, most setae $\beta$-keratins were clustered on a single scaffold (scaffold 426) of the G. japonicus assembly, suggesting regional duplication events. These genomic characteristics may be related to the need for vast abundance of proteins to create the large number of setae in G. japonicus. In the other two species, a total of 16 setae $\beta$-keratin genes were identified in the An. carolinensis genome, and no setae $\beta$-keratin genes were found in the Al. sinensis genome. This result suggested that $\beta$-keratin genes expansion is positively correlated with setae formation in the assessed species. To date the periods of setae $\beta$-keratin expansion in G. japonicus, we established a phylogenetic tree using $133 \beta$-keratin protein sequences from several reptiles and birds ${ }^{24}$, and calculated the divergence value of the branch site in the tree. The timescale of $\beta$-keratin expansion was determined by reference to the timescale of divergence of scale and claw $\beta$-keratins in birds $(\sim 156 \mathrm{Myr}$ ago $)$ and the period of feather keratins expansion (66-51 Myr ago $)^{25,26}$. The results showed that setae $\beta$-keratins experienced twice expansions: one at $105-96 \mathrm{Myr}$ ago and the other at $87-80 \mathrm{Myr}$ ago (Fig. 3b). The expansion period of setae $\beta$-keratin genes proposed by this strategy was very close to the period of setae emergence in gecko indicated by fossil evidence ${ }^{27}$. Of course, the comparative analysis of $\beta$-keratin genes expansion is based on only three genomes, and it is not possible to conclude whether the patterns seen in the G. japonicus genome are unique to this species.

Nocturnal vision adaptation in G. japonicus. As a nocturnal animal, G. japonicus possesses several sensory system characteristics, such as light sensitivity ${ }^{28}$, reduced colour vision, multifocal optical system, high olfaction ${ }^{29}$ and special auditory senses ${ }^{30}$. Collectively, these features improve the ability of G. japonicus to catch prey, evade predators and communicate in low-light environments ${ }^{28}$.

Vertebrate photoreceptor cells are categorized as rods and cones. Rods are responsible for dim-light vision, and cones for daylight and colour vision. Most geckos are nocturnal and possess retinas primarily made up of single and double cones ${ }^{31}$. A premise termed the transmutation theory, proposes that cones in nocturnal geckos were transformed from the cones of some ancestral diurnal lizard ${ }^{32}$. We assessed our genome data for evidence indicating how retinal pigment genes evolved during scotopic adaptation. The photosensitive molecules within photoreceptor cells consist of chromophore and opsin (protein moiety). The visual opsins are classified into five paralogs: rod

Figure 3 | Evolutionary analysis of setae $\boldsymbol{\beta}$-keratins. (a) Phylogenetic tree of $\beta$-keratins from $G$. japonicus. The red branches represent $\beta$-keratins belonging to the primary components of setae, the blue branches represent the components in pad lamella for supporting setae and the green branches represent $\beta$-keratins in scales or claws. A total of $48 \beta$-keratins (purple font) are clustered in scaffold 426 , of these 46 have a single exon. The combinatorial numbers following the keratin names indicate the following protein characteristics: 1: S-core box (SEVTIQPPPCTVVVPGPVLA, sequence similarity $\geq 70 \%$, 35 proteins); 2: cysteine-rich (Cys >10\%, 19 proteins); 3: glycine-rich (Gly >15\%, 36 proteins); 4: isoelectric point ( $p l>7,34$ proteins); 5: molecular weight (Wt $<15,000,58$ proteins); 0 : none of the above. $\beta$-keratins associated with clinging ability have undergone extensive expansion and have higher isoelectric points. (b) Calculation of the expansion period for primary gecko setae $\beta$-keratins using protein sequences from $71 \beta$-keratins of $G$. japonicas (orange), $23 \beta$-keratins of An. carolinensis (light blue), $2 \beta$-keratins of Al. sinensis and $1 \beta$-keratin-like of Crocodylus niloticus (pink), and $36 \beta$-keratins associated with bird's claw, scale and feather (violet, including the following birds: Gallus gallus, Chlamydotis macqueenii, Opisthocomus hoazin, Mesitornis unicolor, Haliaeetus leucocephalus, Leptosomus discolor, Nestor notabilis, Chaetura pelagica, Pterocles gutturalis, Tinamus guttatus, Pygoscelis adeliae, Tauraco erythrolophus, Manacus vitellinus, Picoides pubescens, Mycteria americana, Cathartes aura and Eurypyga helias). The divergence of scale and claw keratins occurs in a birds ancestor $\sim 156 \mathrm{Myr}$ ago, and the feather keratins expansion occurred in birds $\sim 66 \mathrm{Myr}$ ago. $\beta$-keratins in setae of G. japonicus have undergone two expansion periods: one approximately 105-96 Myr ago and the other approximately 87-80 Myr ago. 
pigments RH1 (rhodopsin), cone pigments RH2 (RH1-like), SWS1 (short wavelength-sensitive type 1), SWS2 (SWS1-like) and LWS/MWS (long wavelength-sensitive and middle wavelengthsensitive). We identified nine opsin genes in the genome of
G. japonicus and 20 opsin genes in the genome of $A$. carolinensis (Supplementary Tables 21 and 22), a diurnal lizard with typical tetra chromatic visual system. Our analysis revealed that A. carolinensis possessed a complete set of opsin paralogs, while

a

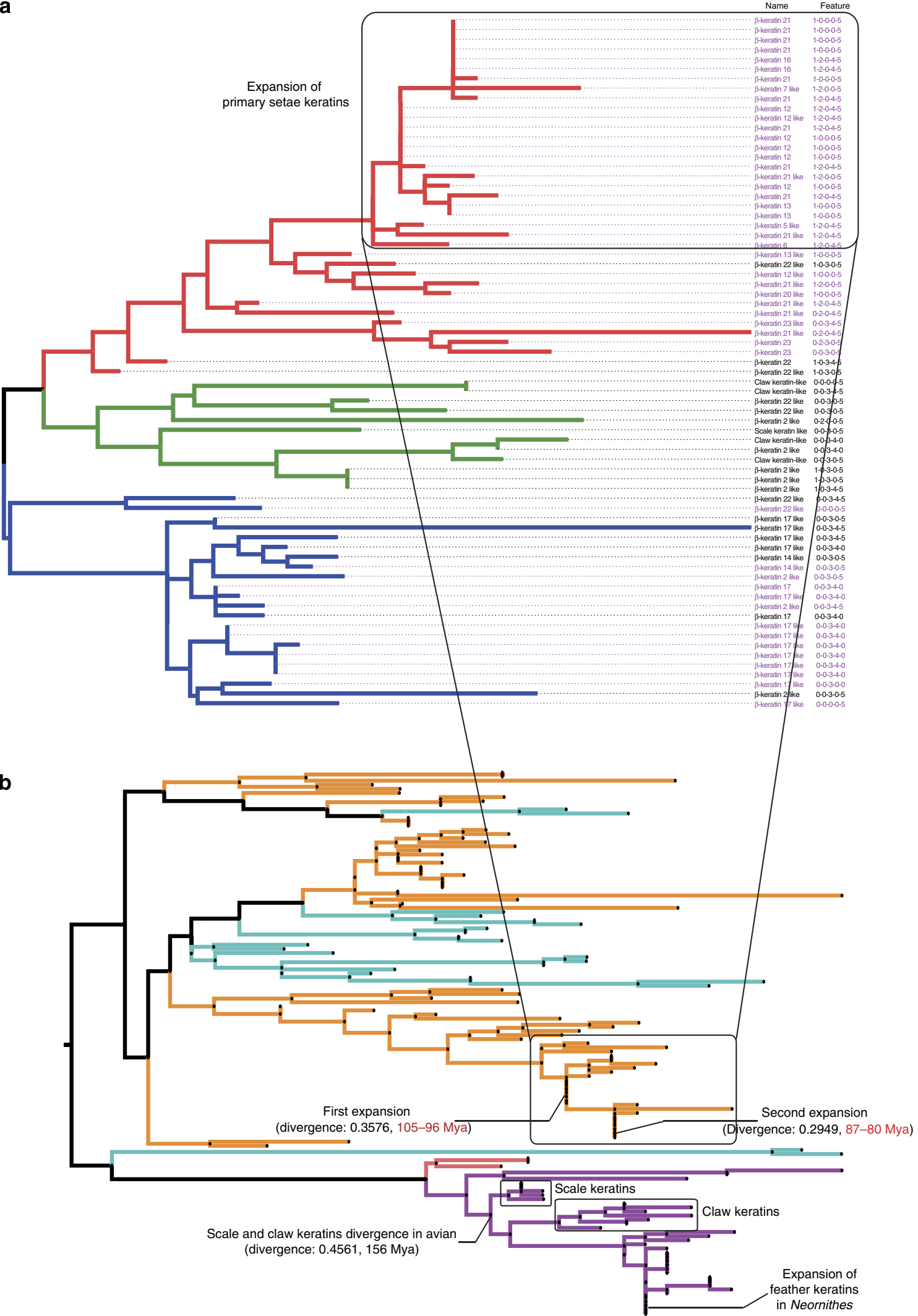

(Divergence: 0.2547, 66-51 Mya) 
a b

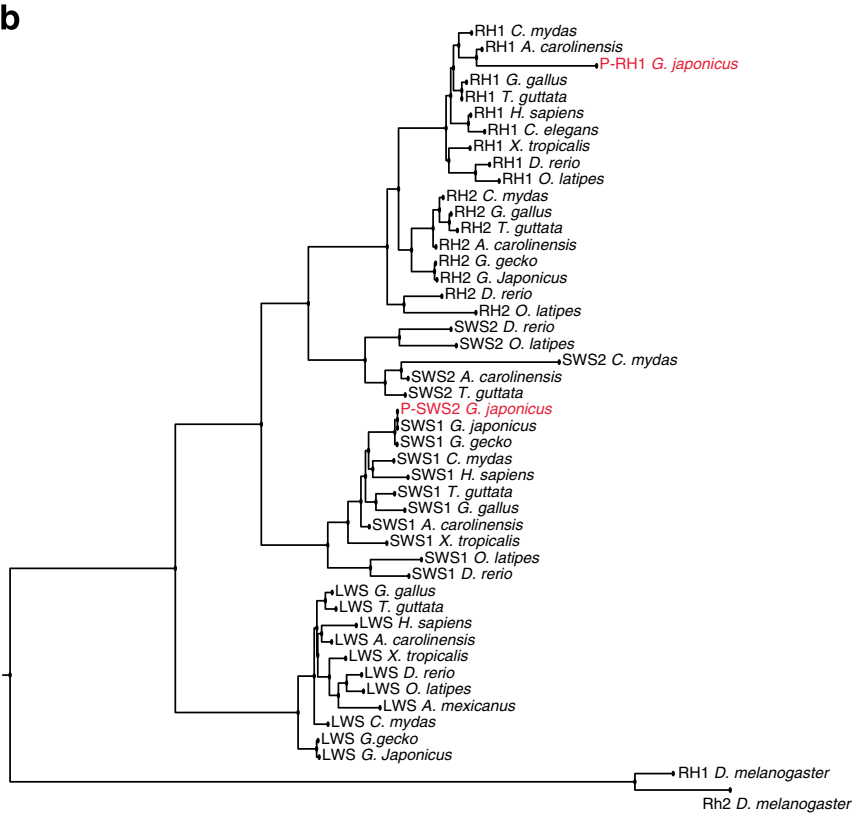

C
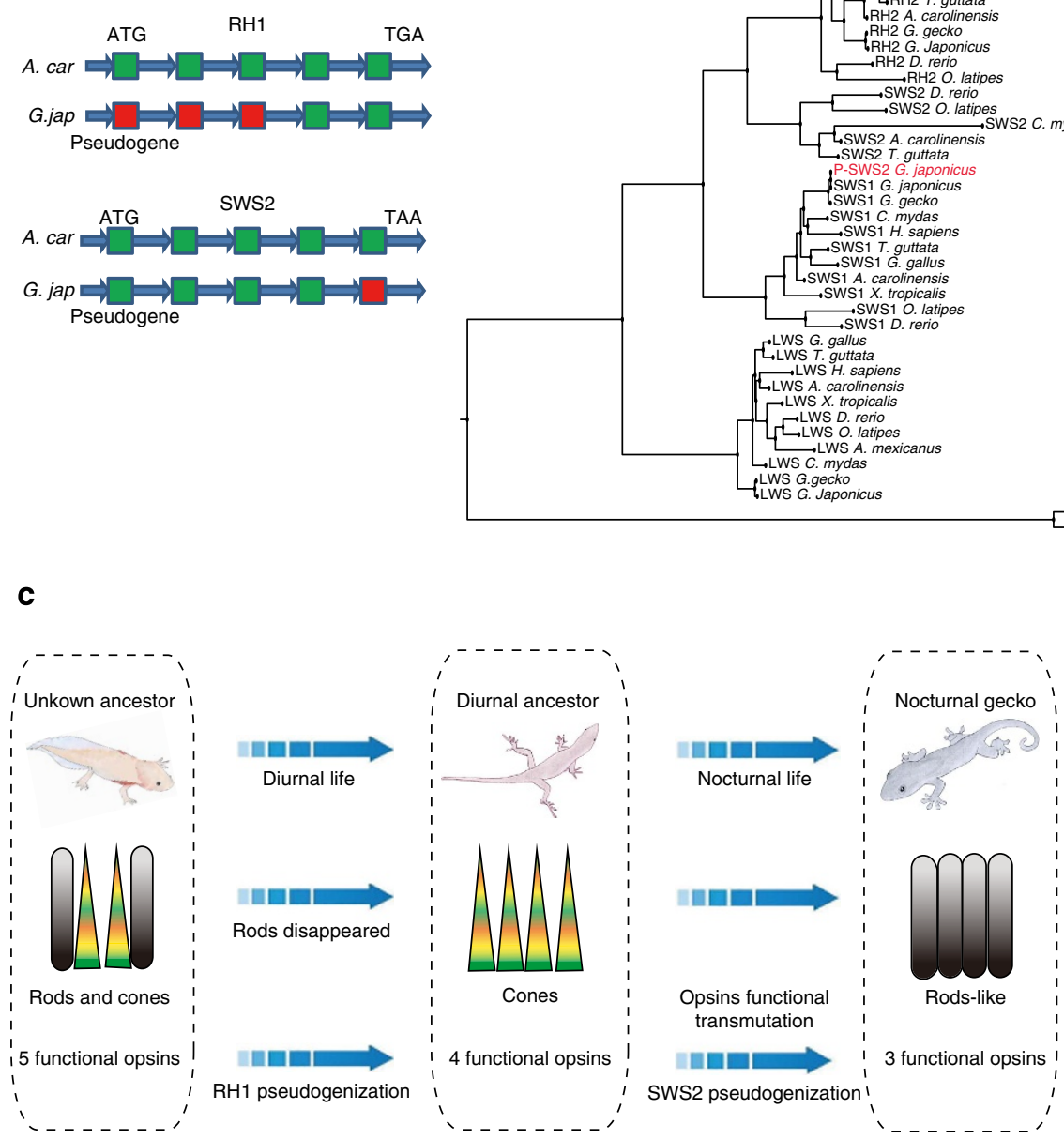

Figure 4 | Opsins genes in the G. japonicus genome. (a) Analysis of RH1 and SWS2 pseudogenes showed mutations in both initiation and termination codons. In addition, the exons of RH1 and SWS2 in G. japonicus were lost, incomplete or shifted in comparison with those in A. carolinensis. The green boxes indicate exons with similarity to those in A. carolinensis. The red boxes represent the lost exons. (b) Phylogenetic analysis of opsin genes from Drosophila melanogaster, Danio rerio, Oryzias latipes, Xenopus tropicalis, Chelonia mydas, Gallus gallus, Taeniopygia guttata, Anolis carolinensis, Gekko gecko and Homo sapiens. The pseudogenes in G. japonicus (P-RH1 and P-SWS2) are indicated in red. (c) Opsin evolution in G. japonicus at genomic level agrees with observed evolutionary variation of retinal cell type and visual sense.

G. japonicus had only three functional opsins: SWS1, LWS and $\mathrm{RH} 2$, all of which are usually found in cones (Supplementary Table 22). In searching for the remaining two opsins, we were able to identify the rod pigment $R H 1$ and the cone pigment SWS2 in G. japonicus, however, they were nonfunctional pseudogenes. The phylogenetic relationship between the opsins form different species is shown in Fig. 4b. We found that RH1 was more divergent between $G$. japonicus and A. carolinensis than was $S W S 2$, indicating that the loss of $R H 1$ occurred earlier than that of SWS2 (Fig. 4a). These results were in agreement with the hypothesis that the ancestors of modern geckos are diurnal lizard without rod opsins ${ }^{28}$. These ancestral species later lost the cone opsin SWS2, potentially as a partial adaptation towards nocturnal vision $^{33}$. Our data provide, for the first time, the evidence of a RH1 deficiency at the genome level in nocturnal geckos.

These data lead to the question of what functional opsins are responsible for nocturnal vision in G. japonicus. Previous reports have suggested that opsin $\mathrm{RH} 2$ amino acid site 89 is an important functional determinant of rod and cone visual pigments ${ }^{33}$. Our comparative analysis indicated that $\mathrm{RH} 2$ in G. japonicus possesses the amino acid replacements F89C, which enables $\mathrm{RH} 2$ to have rod pigment-specific biochemical characteristics ${ }^{32-34}$ (Supplementary Table 23). Our results suggest a possible functional switch or compensatory change in cone pigment of gecko that allows them to receive more light. In conclusion, our results surrounding the evolution of opsin genes at genomic level support the hypothesis that nocturnal G. japonicus evolved from the diurnal ancestors. Furthermore, the pseudogenization and functional switch of opsins is concordant with the variation of retinal cell type, which includes the loss of rods and transformation of cones (Fig. 4c). One thing to note here is that the evolution pattern of opsin we proposed is based on genome data of few species. More data, including genomes of other related species, and phylogenetic analyses are necessary to test this hypothesis and to determine how and when particular changes occurred in the opsin families. 
Diversified olfaction of G. japonicus. Improved survival in a low-light environment typically includes the evolution of more sensitive sense of smell to better obtain food and safety ${ }^{35}$. Our analysis of expanded gene families in 16 species revealed that G. japonicus had a significant expansion of olfactory receptor (OR) genes. OR genes are divided into Class I and Class II. The Class I genes encode odour receptors that detect molecules in water, and can be divided into the following sub-groups: $\alpha, \beta, \varepsilon, \zeta$ and $\delta$. The class II genes encode odour receptors that detect molecules in air, and only have a $\gamma$ sub-group ${ }^{36}$. According to our gene family analysis, G. japonicus displayed more diversity of $O R$ genes $(\alpha-, \beta$ - and $\gamma$-ORs) than any of other assessed reptiles and more than even human $(\alpha-, \gamma$-ORs). Usually, a single air OR can only identify one or a few scents ${ }^{37}$, thus, the presence of a higher number of ORs may indicate greater diversity in odour sensing. To investigate the types and numbers of ORs in species with different features, ORs from seven species (G. japonicus, $X$. tropicalis, D. rerio, An. carolinensis, H. sapiens, Al. sinensis and T. rubripes) were compared. Because the assembly of ORs is difficult, then the assembly quality of these genomes is a notable

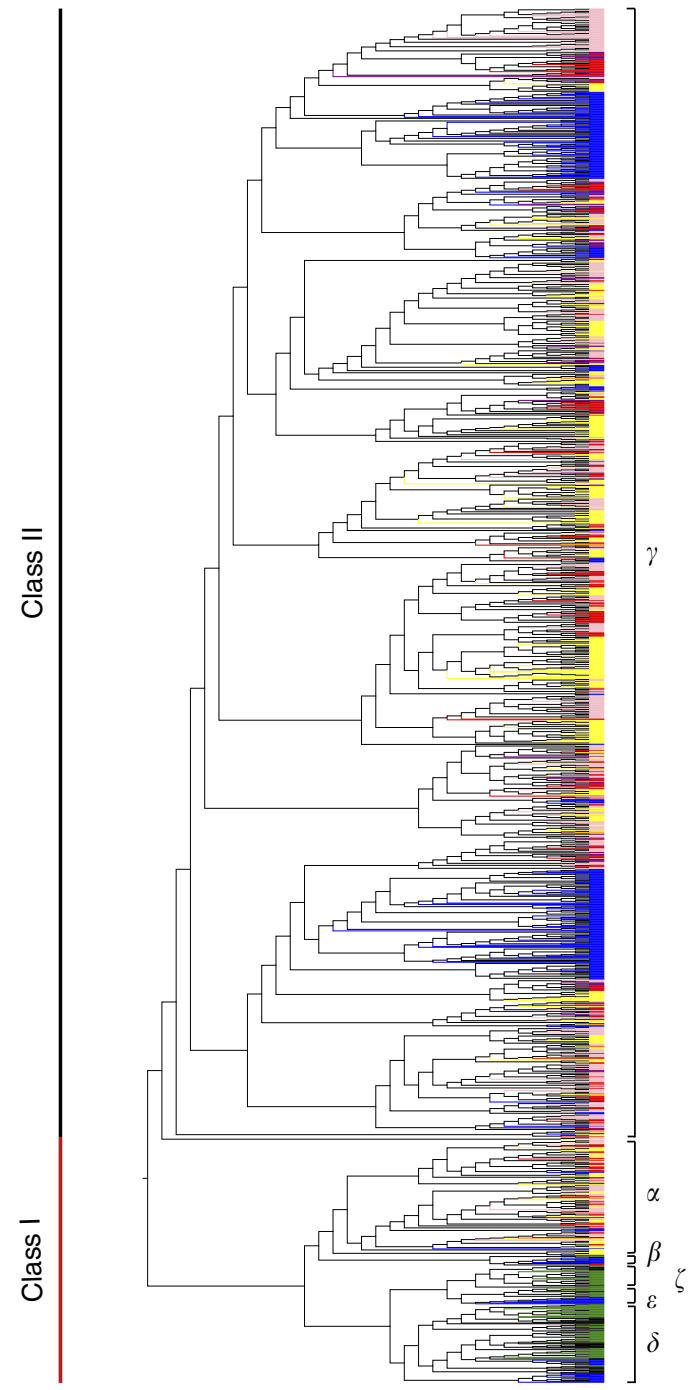

Figure 5 | Phylogenetic tree of functional $O R$ genes in seven species. The Class I genes (including $\alpha, \beta, \varepsilon, \zeta$ and $\delta$ ORs) encode proteins used for scent detection in water. The Class II gene $(\gamma-O R)$ encode proteins used for scent detectionin air. G. japonicus has undergone extensive expansion in Class II genes. Red, G. japonicus; blue, $X$. tropicalis; green, D. rerio; purple, An. carolinensis; yellow, H. sapiens; pink, Al. sinensis; black, T. rubripes. concern for the comparative analysis of ORs. The parameters associated with the genome assembly quality are listed in Supplementary Table 24 . The comparative results show that the number of OR genes in G. japonicus (251) is almost three times that in A. carolinensis, a diurnal relative of G. japonicus (87) (Fig. 5, Supplementary Table 25). Furthermore, nocturnal G. japonicus had many functional $\gamma$-ORs for detecting airborne chemicals, which may serve to complement visual senses when seeking prey ${ }^{33,38}$. The ability to detect airborne chemicals has been shown to contribute to survival in nocturnal lizards and birds $^{38,39}$. Likewise, the substantial expansion of $O R$ genes for scenting airborne odorants in geckos might improve their nighttime survival.

Positive selection for tail regeneration in G. japonicus. Geckos can detach their tails when they are attacked, and this adaptive physiological process has evolved in many saurian animals to enable quick escape from predators. On tail detachment, multiple tissue regeneration pathways initiate some conserved repair processes including wound healing ${ }^{40}$, blastema formation and tissue remodelling ${ }^{1-43}$. Following this, a new tail will grow within a few months. We searched the G. japonicus genome for genomic regions showing positive selection for tail regeneration in G. japonicus, as such genes are likely important to this process. To identify PSGs, single-copy orthologs were selected from six reptile gene families (G. japonicus, An. carolinensis, Al. sinensis, C. mydas, Pelodiscus sinensis and P. bivittatus). Then, we carried out multiple alignments of the single-copy orthologs and calculated the $\mathrm{Ka} / \mathrm{Ks}$ ratios to identify PSGs in three reptile species (G. japonicus and An. carolinensis two species with tail regeneration ability, as well as Al. sinensis, a species without this ability). We obtained 155, 178 and 171 PSGs from G. japonicus, An. carolinensis and Al. sinensis, respectively (Supplementary Table 26). Gene Ontology annotation revealed that some PSGs of G. japonicus were likely related to regenerative abilities, as these PSGs were enriched in the categories of wound healing, tissue regeneration, cell proliferation or migration, prostaglandin biosynthetic process and other relevant categories (Fig. 6a). These biological processes are essential for successful regeneration, as reported by many studies on limb and tail regeneration in other species ${ }^{40-44}$. The PSGs in A. carolinensis and G. japonicus contain several shared Gene Ontology terms, such as cell proliferation and prostaglandin biosynthetic process. It is notable that prostacyclin synthase (PTGIS) and prostaglandin-endoperoxide synthase 1 (PTGS1), which are involved in prostaglandin biosynthesis, were under positive selection in both G. japonicus and A. carolinensis. This indicates the likelihood that these genes are involved in similar biological processes that may promote tail regeneration after injury. To investigate whether the PSGs in G. japonicus had activity during tail regeneration, we collected transcriptome data from regenerating stump tissue at different time points following tail amputation. The results showed that $69 \%$ of the PSGs were upregulated at 1 day, 3 days or 7 days after autotomy (Fig. 6b). Among these genes, the expression of both PTGIS and PTGS1, which encode key enzymes in prostaglandin synthesis, increased by 3 days after tail amputation. Prostanoids have been reported to be involved in the regeneration of various tissues and organs, including liver, muscle, nerve and tail in different species ${ }^{45-48}$ (Fig. 6c). These findings support the hypothesis that PSGs may be involved in tail regeneration or other adaptive physiological processes in G. japonicus.

\section{Discussion}

Gecko is an important clade of reptile that possesses several amazing abilities, such as the ability to cling at vertical surface 
a

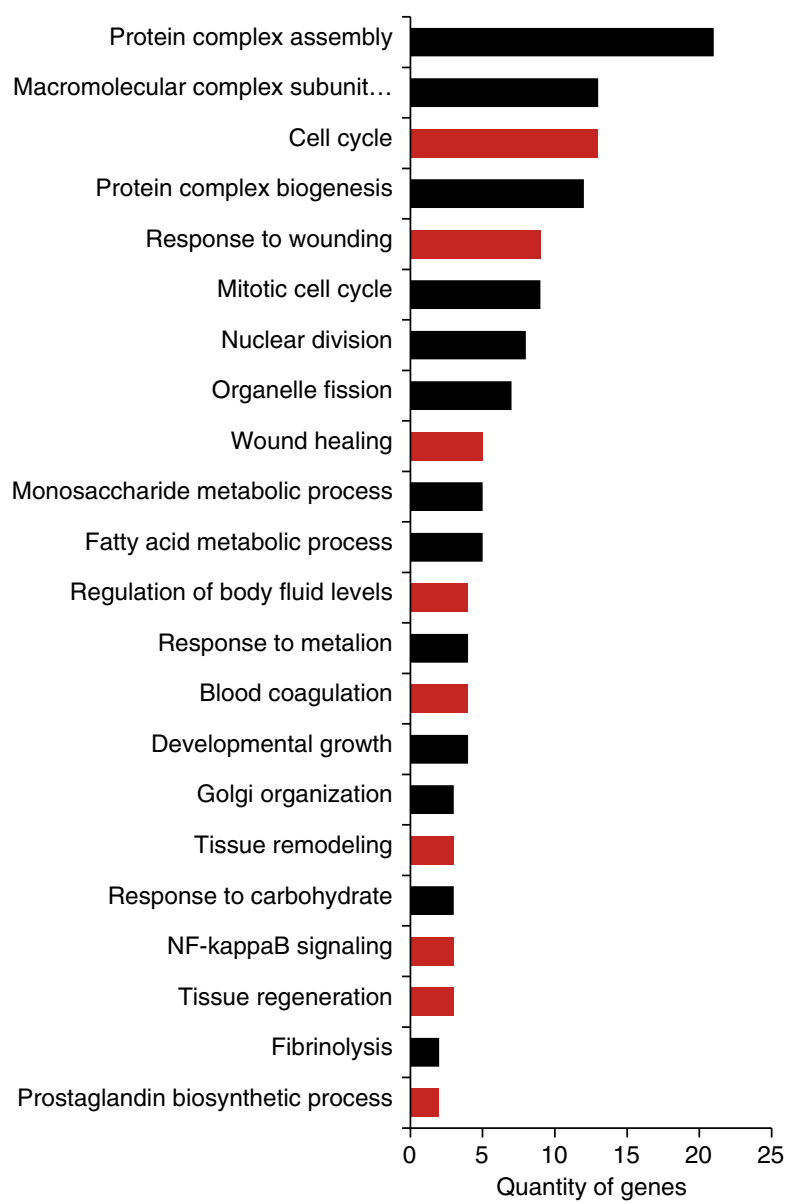

b

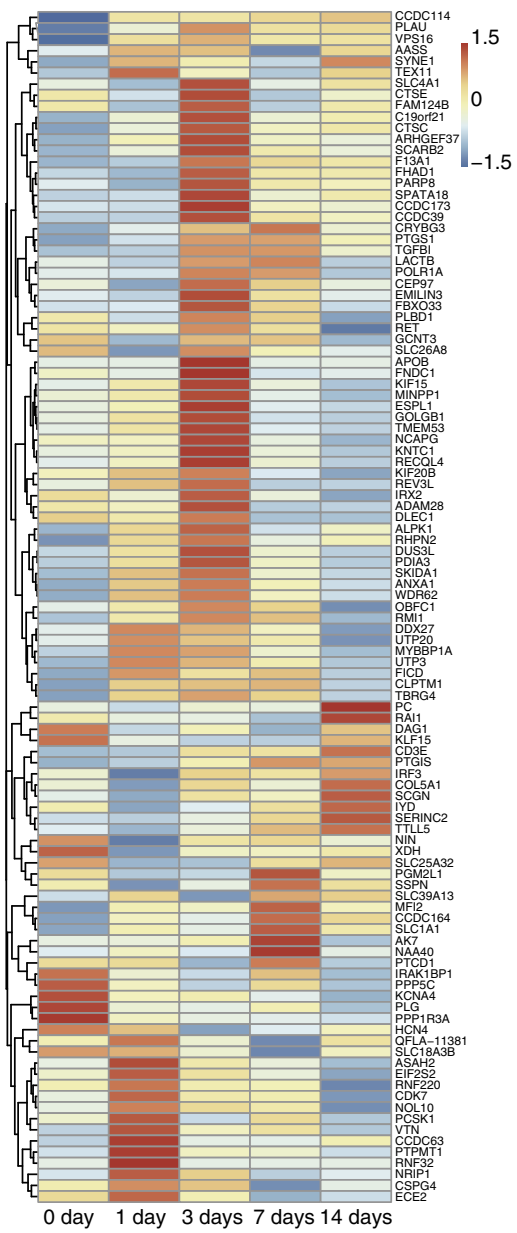

C

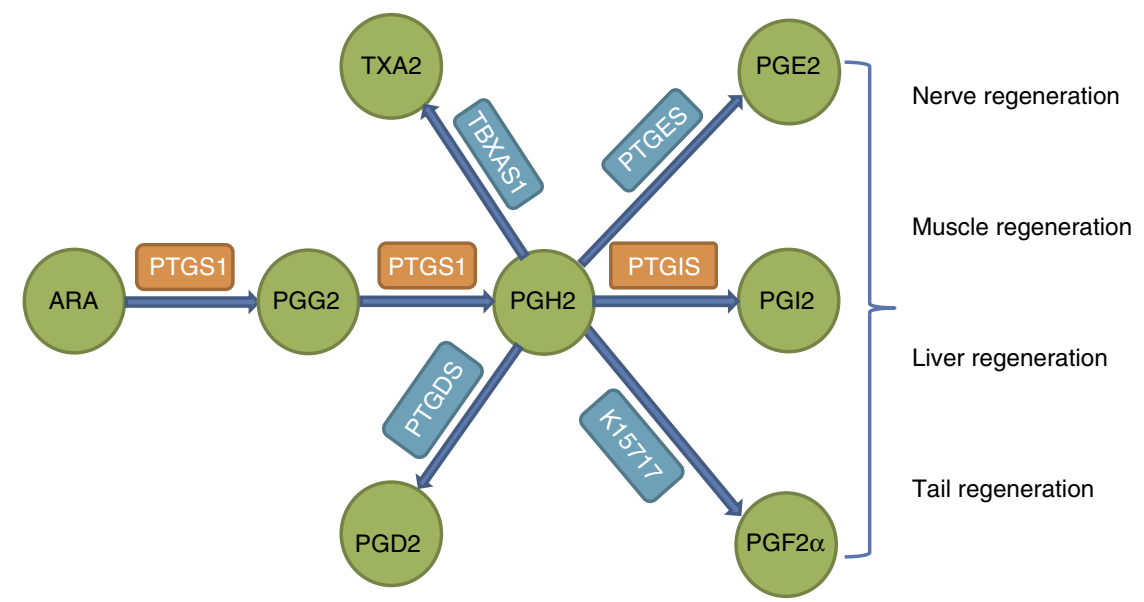

Figure 6 | Positively selected genes (PSGs) related to tail regeneration in G. japonicus. (a) Analysis of 155 PSGs in G. japonicus based on representative gene ontology biological processes. Categories with red bars such as cell cycle (GO:0007049), response to wounding (GO:0009611), wound healing (GO:0042060), tissue regeneration (GO:0042246), tissue remodelling (GO:0048771), blood coagulation (GO:0007596) and prostaglandin biosynthetic process (GO:0001516) are likely to be involved in tail regeneration after autotomy. (b) Heatmap of 107 PSGs in G. japonicus at different time points following tail autotomy. Approximately $70 \%$ of the PSGs were detected in transcriptome data. (c) Pathway of arachidonic acid (ARA) metabolism in G. japonicus. The circles with green backgrounds represent ARA and its derivatives. The boxes with orange backgrounds show the key synthases under positive selection in $\mathrm{G}$. japonicus genome. The boxes with blue backgrounds show the normal synthases in $\mathrm{G}$. japonicus genome.

and tail regeneration. These characteristics have been extensively investigated for many years. However, the absence of genomic data for Gekkonidae species hinders the mechanism study underlying these interesting phenomena. In this study we acquired the genome sequence of $G$. japonicus and used this information to investigate the genetic basis of many behavioural and physiological characteristics of geckos by searching for key genes potentially involved in clinging ability, low-light visual 
activity, highly developed olfaction and regenerative ability. Although our study cannot be considered as an in-depth analysis at the present stage, it provides a foundation for future mechanistic studies, particularly with regard to regeneration. We identified potential candidate genes that might contribute to the regeneration process using genome comparative analysis. Such candidates include PTGIS and PTGS1, which are involved in prostaglandin synthesis. Prostaglandin metabolism was recently reported to be tightly associated with regeneration in multiple tissues ${ }^{49}$. The timing and manner of the involvement of these genes in tail regeneration of G. japonicus is an attractive topic for further study. In addition, gecko species can also serve as an important model for the studies of sex determination ${ }^{50}$ and reproductive strategies ${ }^{51}$, for the reasons that these species situate on the nodes switching environment sex determination to genetic sex determination, oviparity pattern to viviparity pattern. The G. japonicus genomic data obtained in the current study will be of great value in studying these essential evolutionary events.

\section{Methods}

G. japonicus sample. The G. japonicus used in this study were sampled in Jiangsu, China. The adult geckos were freely fed mealworms and given water during the whole experiment. All experimental protocols pertinent to animals were given before approval by the Laboratory Animal Care and Use Committee of the Nantong University.

Genome sequencing and assembly. Genomic DNA was extracted from tissues of an adult male G. japonicus. The DNA was fragmented and the fragments were purified by electrophoresis for whole-genome sequencing. DNA Libraries were constructed according to the 'Mate Pair Library v2 Sample Preparation Guide for $2-5 k b$ Libraries' and 'Paired-End Sample Preparation Guide' from Illumina. PCR amplification was performed following the addition of adaptors, and the products were clustered for the mate-pair libraries (insert size $\geq 2 \mathrm{~Kb}$ ). The genomic DNA was sequenced using Illumina Hiseq2000 (20 lanes, $330.90 \mathrm{~Gb}, 131.35 \times$ ). Raw data were generated, after filtering $233.49 \mathrm{~Gb}$ of clean data remained for de novo assembly. Whole-genome assembly was performed using SOAPdenovo ${ }^{52}$ and SSPACE software ${ }^{33}$. Furthermore, 10 fosmid clones were subjected to Sanger sequencing and were used as reference data to ensure genomic coverage.

Genome annotation and evolution. Repeats in DNA sequence of G. japonicus were characterized by homologue-based identification using RepeatMasker (http:// www.repeatmasker.org) and Repbase ${ }^{54}$. Repeated proteins were marked using RepeatProteinMask(http://www.repeatmasker.org), and de novo interspersed repeat annotation was performed using RepeatModeler (http://www.repeatmasker. org/RepeatModeler.html). An extra RepeatMasker analysis was applied after de novo identifications of repeats. In addition, tandem repeats were identified using Tandem Repeat Finder ${ }^{55}$. Gene prediction was created by GLEAN (http:// sourceforge.net/projects/glean-gene) integration of de novo and homologous gene models. RNA-seq data were subsequently used to refine the gene set. De novo prediction was performed based on the repeat-masked genome. Two programs AUGUSTUS ${ }^{56}$ and GENSCAN ${ }^{57}$ were applied in the prediction. The homologuebased prediction included the mapping of protein sequences (downloaded from NCBI) of closely related representative species An. carolinensis, Gallus gallus, Homo sapiens, Meleagris gallopavo and Xenopus tropicalis to the genome using TblastN, aligning and searching for accurate spliced alignments using GeneWise ${ }^{58}$. The EST of G. japonicus (downloaded from NCBI) was aligned against the assembled genome using BLAT to generate spliced alignments, and PASA was used to filter the overlapping sequences to link the spliced alignments and predict the possible gene model. Evidences were integrated by GLEAN to produce a consensus gene set. In addition, transcriptomes of multiple tissues were aligned to the genome using TopHat $^{59}$. These were assembled using Cufflinks ${ }^{60}$ to improve the accuracy and completeness of the predicted gene set. To conclude gene functions, we scanned the final gene set with $\mathrm{KEGG}^{61}$, SwissProtand TrEMBL ${ }^{62}$. InterProScan was also used to confirm motifs and domains in the final gene set of G. japonicus ${ }^{63}$.

Gene family expansion. We identified gene family expansion and contraction using CAFE ${ }^{64}$, which employed a random birth and death model to study gene gain and loss in gene families across a user-specified phylogeny. The global parameter $\lambda$, which described both the gene birth $(\lambda)$ and death $(\mu=-\lambda)$ rate across all branches in the tree for all gene families, was estimated using maximum likelihood. A conditional $P$ value was calculated for each gene family, and families with conditional $P$ values less than threshold $(0.0001)$ were considered to have accelerated rates of gain or loss. We identified branches that were responsible for low overall $P$ values of significant families.
Phylogenetic analyses. We constructed a phylogenetic tree that included Alligator sinensis, Anolis carolinensis, Pelodiscus sinensis, Chelonia mydas, Xenopus tropicalis, Canis familiaris, Oryzias latipes, Homo sapiens, Meleagris gallopavo, Gallus gallus, Ornithorhynchus anatinus, Danio rerio, Taeniopygia guttata, Python molurus bivittatus and G. japonicus using 696 single-copy orthologous genes from gene family construction. Each of the orthologous genes was subjected to multiple sequence alignment with Muscle ${ }^{65}$ and concatenated into a super sequence. PhyML $^{66}$ was used to construct the phylogenetic tree under the GTR and Inv- $\gamma$ model functions. The same sequence set was applied to estimate the periods of species divergence using the program PAML MCMCTREE under correlated molecular clock function in the approximate likelihood calculation method ${ }^{67}$. The correlated molecular clock and REV substitution model were selected to perform estimation. The MCMC process of PAML mcmctree was run to sample 100,000 times with a sample frequency of 50, after a burn-in of 5,000,000 iterations. Fossil calibrates were derived from http://www.fossilrecord.net/dateaclade/index.html.

Positively selected genes. We calculated $\mathrm{Ka} / \mathrm{Ks}$ ratios for all single-copy orthologs of G. japonicus, Anolis carolinensis, Alligator sinensis, Python molurus bivittatus, Chelonia mydas and Pelodiscus sinensis. Alignment quality was essential for estimating positive selection. Thus, orthologous genes were first aligned using PRANK $^{68}$, a favored alignment tool for molecular evolution studies ${ }^{69}$. We used Gblocks to remove ambiguously aligned blocks within the PRANK alignments ${ }^{70}$. We employed 'codeml' in the PAML package with the free-ratio model to estimate $\mathrm{Ka}, \mathrm{Ks}$ and $\mathrm{Ka} / \mathrm{Ks}$ ratios on different branches. The differences in the mean $\mathrm{Ka} / \mathrm{Ks}$ ratios for single-copy genes between G. japonicus and each of the other species were compared with paired Wilcoxon rank-sum tests. After filtering out the false positive, we obtained a final total of 155 positive selection genes in G. japonicus.

\section{References}

1. Wang, D. Y., Kumar, S. \& Hedges, S. B. Divergence time estimates for the early history of animal phyla and the origin of plants, animals and fungi. Proc. Biol. Sci. 266, 163-171 (1999).

2. Peter, U. The original descriptions of reptiles. Zootaxa 2334, 59-68 (2010).

3. Torstrom, S. M., Pangle, K. L. \& Swanson, B. J. Shedding subspecies: the influence of genetics on reptile subspecies taxonomy. Mol. Phylogenet. Evol. 76, 134-143 (2014).

4. Vidal, N. \& Hedges, S. B. The phylogeny of squamate reptiles (lizards, snakes, and amphisbaenians) inferred from nine nuclear protein-coding genes. C. R. Biol. 328, 1000-1008 (2005).

5. Wever, E. G. The lizard ear: Gekkonidae. J. Morphol. 143, 121-165 (1974).

6. Fleming, P. A., Valentine, L. E. \& Bateman, P. W. Telling tails: selective pressures acting on investment in lizard tails. Physiol. Biochem. Zool. 86, 645-658 (2013).

7. Autumn, K. et al. Adhesive force of a single gecko foot-hair. Nature 405, 681-685 (2000).

8. Autumn, K. et al. Evidence for van der Waals adhesion in gecko setae. Proc. Natl Acad. Sci. USA 99, 12252-12256 (2002).

9. McLean, K. E. \& Vickaryous, M. K. A novel amniote model of epimorphic regeneration: the leopard gecko, Eublepharis macularius. BMC Dev. Biol. 11, 50 (2011).

10. Boesel, L. F., Greiner, C., Arzt, E. \& del Campo, A. Gecko-inspired surfaces: a path to strong and reversible dry adhesives. Adv. Mater. 22, 2125-2137 (2010).

11. Castoe, T. A. et al. The Burmese python genome reveals the molecular basis for extreme adaptation in snakes. Proc. Natl Acad. Sci. USA 110, 20645-20650 (2013)

12. Wan, Q. H. et al. Genome analysis and signature discovery for diving and sensory properties of the endangered Chinese alligator. Cell Res. 23, 1091-1105 (2013).

13. Wang, Z. et al. The draft genomes of soft-shell turtle and green sea turtle yield insights into the development and evolution of the turtle-specific body plan. Nat. Genet. 45, 701-706 (2013).

14. Alfoldi, J. et al. The genome of the green anole lizard and a comparative analysis with birds and mammals. Nature 477, 587-591 (2011).

15. Green, R. E. et al. Three crocodilian genomes reveal ancestral patterns of evolution among archosaurs. Science 346, 1254449 (2014).

16. Tempel, S. Using and understanding RepeatMasker. Methods Mol. Biol. 859, 29-51 (2012)

17. Daza, J. D., Bauer, A. M. \& Snively, E. D. On the fossil record of the Gekkota. Anat. Rec. (Hoboken) 297, 433-462 (2014).

18. Tarling, D. H. Gondwanaland, palaeomagnetism and continental drift. Nature 229, 17-21 71 (1971)

19. Pyron, R. A., Burbrink, F. T. \& Wiens, J. J. A phylogeny and revised classification of Squamata, including 4161 species of lizards and snakes. BMC Evol. Biol. 13, 93 (2013).

20. Rodriguez-Marco, N. A., Larrea-Goni, N. \& Solanas-Alava, S. Prehistoric eyes: Ophthalmosaurus and the Tuatara. Arch. Soc. Esp. Oftalmol. 88, e33-e34 (2013). 
21. Magin, T. M., Vijayaraj, P. \& Leube, R. E. Structural and regulatory functions of keratins. Exp. Cell Res. 313, 2021-2032 (2007).

22. Alibardi, L. Immunolocalization of keratin-associated beta-proteins (beta-keratins) in pad lamellae of geckos suggest that glycine-cysteine-rich proteins contribute to their flexibility and adhesiveness. J. Exp. Zool. A Ecol. Genet. Physiol. 319, 166-178 (2013).

23. Alibardi, L. Cell biology of adhesive setae in gecko lizards. Zoology (Jena) 112, 403-424 (2009).

24. Ye, C., Wu, X., Yan, P. \& Amato, G. beta-Keratins in crocodiles reveal amino acid homology with avian keratins. Mol. Biol. Rep. 37, 1169-1174 (2010).

25. Zhang, G. et al. Comparative genomics reveals insights into avian genome evolution and adaptation. Science 346, 1311-1320 (2014).

26. Greenwold, M. J. \& Sawyer, R. H. Linking the molecular evolution of avian beta (beta) keratins to the evolution of feathers. J. Exp. Zool. B Mol. Dev. Evol. 316, 609-616 (2011).

27. Arnold, E. N \& Poinar, G. O. A 100 million year old gecko with sophisticated adhesive toe pads, preserved in amber from Myanmar. Zootaxa 1847, 62-68 (2008).

28. Roll, B. Gecko vision-visual cells, evolution, and ecological constraints. J. Neurocytol. 29, 471-484 (2000).

29. Schwenk, K. The evolution of chemoreception in squamate reptiles: a phylogenetic approach. Brain. Behav. Evol. 41, 124-137 (1993).

30. Bergevin, C. Comparison of otoacoustic emissions within gecko subfamilies: morphological implications for auditory function in lizards. J. Assoc. Res. Otolaryngol. 12, 203-217 (2011).

31. Roth, L. S., Lundstrom, L., Kelber, A., Kroger, R. H. \& Unsbo, P. The pupils and optical systems of gecko eyes. J. Vis. 9, 27.1-11 (2009).

32. Kojima, D. et al. Cone visual pigments are present in gecko rod cells. Proc. Natl Acad. Sci. USA 89, 6841-6845 (1992).

33. Yokoyama, S. \& Blow, N. S. Molecular evolution of the cone visual pigments in the pure rod-retina of the nocturnal gecko, Gekko gekko. Gene 276, 117-125 (2001).

34. Yokoyama, S. \& Tada, T. Evolutionary dynamics of rhodopsin type 2 opsins in vertebrates. Mol. Biol. Evol. 27, 133-141 (2010).

35. Tonosaki, K. \& Shibuya, T. Action of some drugs on gecko olfactory bulb mitral cell responses to odor stimulation. Brain Res. 167, 180-184 (1979).

36. Niimura, Y. Evolutionary dynamics of olfactory receptor genes in chordates: interaction between environments and genomic contents. Hum. Genomics 4, 107-118 (2009).

37. Hoover, K. C. Evolution of olfactory receptors. Methods Mol. Biol. 1003 , 241-249 (2013).

38. Gordon, C. E., Dickman, C. R. \& Thompson, M. B. What factors allow opportunistic nocturnal activity in a primarily diurnal desert lizard (Ctenotus pantherinus)? Comp. Biochem. Physiol. A Mol. Integr. Physiol. 156, 255-261 (2010).

39. Steiger, S. S., Fidler, A. E. \& Kempenaers, B. Evidence for increased olfactory receptor gene repertoire size in two nocturnal bird species with well-developed olfactory ability. BMC Evol. Biol. 9, 117 (2009).

40. Hutchins, E. D. et al. Transcriptomic analysis of tail regeneration in the lizard Anolis carolinensis reveals activation of conserved vertebrate developmental and repair mechanisms. PLoS ONE 9, e105004 (2014).

41. Delorme, S. L., Lungu, I. M. \& Vickaryous, M. K. Scar-free wound healing and regeneration following tail loss in the leopard gecko, Eublepharis macularius. Anat. Rec. (Hoboken) 295, 1575-1595 (2012).

42. Love, N. R. et al. Amputation-induced reactive oxygen species are required for successful Xenopus tadpole tail regeneration. Nat. Cell Biol. 15, 222-228 (2013).

43. Niethammer, P., Grabher, C., Look, A. T. \& Mitchison, T. J. A tissue-scale gradient of hydrogen peroxide mediates rapid wound detection in zebrafish. Nature 459, 996-999 (2009).

44. Kimura, Y. et al. Expression of complement 3 and complement 5 in newt limb and lens regeneration. J. Immunol. 170, 2331-2339 (2003).

45. Rudnick, D. A., Perlmutter, D. H. \& Muglia, L. J. Prostaglandins are required for CREB activation and cellular proliferation during liver regeneration. Proc. Natl Acad. Sci. USA 98, 8885-8890 (2001).

46. Hsueh, Y. C., Wu, J. M., Yu, C. K., Wu, K. K. \& Hsieh, P. C. Prostaglandin E(2) promotes post-infarction cardiomyocyte replenishment by endogenous stem cells. EMBO Mol. Med. 6, 496-503 (2014).

47. Kogawa, S., Yasuda, H., Terada, M., Maeda, K. \& Kikkawa, R. Apoptosis and impaired axonal regeneration of sensory neurons after nerve crush in diabetic rats. Neuroreport 11, 663-667 (2000).

48. Sharma, P. \& Suresh, S. Influence of COX-2-induced PGE2 on the initiation and progression of tail regeneration in Northern House Gecko, Hemidactylus flaviviridis. Folia Biol. (Praha) 54, 193-201 (2008).

49. Zhang, Y. et al. TISSUE REGENERATION. Inhibition of the prostaglandindegrading enzyme 15-PGDH potentiates tissue regeneration. Science 348, aaa2340 (2015).

50. Gamble, T. A review of sex determining mechanisms in geckos (Gekkota: Squamata). Sex. Dev. 4, 88-103 (2010).
51. Pyron, R. A. \& Burbrink, F. T. Early origin of viviparity and multiple reversions to oviparity in squamate reptiles. Ecol. Lett. 17, 13-21 (2014).

52. Li, R. et al. De novo assembly of human genomes with massively parallel short read sequencing. Genome Res. 20, 265-272 (2010).

53. Boetzer, M., Henkel, C. V., Jansen, H. J., Butler, D. \& Pirovano, W. Scaffolding pre-assembled contigs using SSPACE. Bioinformatics 27, 578-579 (2011).

54. Jurka, J. et al. Repbase Update, a database of eukaryotic repetitive elements. Cytogenet. Genome Res. 110, 462-467 (2005).

55. Benson, G. Tandem repeats finder: a program to analyze DNA sequences. Nucleic Acids Res. 27, 573-580 (1999).

56. Stanke, M. \& Morgenstern, B. AUGUSTUS: a web server for gene prediction in eukaryotes that allows user-defined constraints. Nucleic Acids Res. 33, W465-W467 (2005).

57. Burge, C. B. \& Karlin, S. Finding the genes in genomic DNA. Curr. Opin. Struct. Biol. 8, 346-354 (1998).

58. Birney, E., Clamp, M. \& Durbin, R. GeneWise and Genomewise. Genome Res. 14, 988-995 (2004).

59. Trapnell, C., Pachter, L. \& Salzberg, S. L. TopHat: discovering splice junctions with RNA-Seq. Bioinformatics 25, 1105-1111 (2009).

60. Trapnell, C. et al. Differential gene and transcript expression analysis of RNA-seq experiments with TopHat and Cufflinks. Nat. Protoc. 7, 562-578 (2012).

61. Wixon, J. \& Kell, D. The Kyoto encyclopedia of genes and genomes-KEGG. Yeast 17, 48-55 (2000).

62. Boeckmann, B. et al. The SWISS-PROT protein knowledgebase and its supplement TrEMBL in 2003. Nucleic Acids Res. 31, 365-370 (2003).

63. Quevillon, E. et al. InterProScan: protein domains identifier. Nucleic Acids Res. 33, W116-W120 (2005).

64. De Bie, T., Cristianini, N., Demuth, J. P. \& Hahn, M. W. CAFE: a computational tool for the study of gene family evolution. Bioinformatics 22, 1269-1271 (2006).

65. Edgar, R. C. MUSCLE: multiple sequence alignment with high accuracy and high throughput. Nucleic Acids Res. 32, 1792-1797 (2004).

66. Guindon, S. et al. New algorithms and methods to estimate maximumlikelihood phylogenies: assessing the performance of PhyML 3.0. Syst. Biol. 59, 307-321 (2010).

67. Yang, Z. PAML: a program package for phylogenetic analysis by maximum likelihood. Comput. Appl. Biosci. 13, 555-556 (1997).

68. Loytynoja, A. \& Goldman, N. Phylogeny-aware gap placement prevents errors in sequence alignment and evolutionary analysis. Science 320, 1632-1635 (2008).

69. Markova-Raina, P. \& Petrov, D. High sensitivity to aligner and high rate of false positives in the estimates of positive selection in the 12 Drosophila genomes. Genome Res. 21, 863-874 (2011).

70. Talavera, G. \& Castresana, J. Improvement of phylogenies after removing divergent and ambiguously aligned blocks from protein sequence alignments. Syst. Biol. 56, 564-577 (2007).

\section{Acknowledgements}

This study was supported by the National Natural Science Foundation of China (Grant Nos. 81130080, 31471011, 31472004, 31171405 and 31071874), the Ministry of Science and Technology of China (973 Programme, 2014 CB542202) and the Priority Academic Program Development (PAPD) of Jiangsu Higher Education Institutions. We also thank Dr Laurie Goodman for his help in manuscript preparation.

\section{Author contributions}

X.G. and H.Y. managed the project and coordinated the work. Q.Z., L.L., L.Y., Y.L., Y.Z., J.L., Z.H. and J.Y. performed genome sequencing, assembly and annotation. Y.L., J.Y., Y.W., J.J. and M.L. performed the genome phylogenentic analysis. Y.L., Y.W., J.Y., B.Y. and Y.L. performed the comparative analysis of $\beta$-keratin genes. Y.L., Y.W., J.Y.,Q.Z.,C.Z. and Y.W. conducted the phylogenetic analysis of opsin genes. Y.L., Y.W., J.Y., M.L., Y.Y., F.D. and L.Y. conducted comparative analysis of olfactory receptors. Y.L., Y.W., J.Y., M.L., Q.Z., L.L. and Y.L. performed the tail regeneration-related PSG analysis and transcriptome analysis. T.Q., M.L., Y.G. and M.X. prepared DNA and RNA sample. J.Y., Y.L., Y.W., Q.Z., X. G. and H.Y. wrote and revised the manuscript.

\section{Additional information}

Accession codes: This Whole Genome Shotgun project of Gekko japonicus has been deposited at DDBJ/EMBL/GenBank under the accession LNDG00000000. The version described in this paper is version LNDG01000000. The RNA-Seq data of Gekko japonicus have been deposited in NCBI database under the accession code SRA304902.

Supplementary Information accompanies this paper at http://www.nature.com/ naturecommunications

Competing financial interests: The authors declare no competing financial interests. 
Reprints and permission information is available online at http://npg.nature.com/ reprintsandpermissions/

How to cite this article: Liu, Y. et al. Gekko japonicus genome reveals evolution of adhesive toe pads and tail regeneration. Nat. Commun. 6:10033 doi: 10.1038/ncomms10033 (2015). (c) (i) This work is licensed under a Creative Commons Attribution 4.0 International License. The images or other third party material in this article are included in the article's Creative Commons license, unless indicated otherwise in the credit line; if the material is not included under the Creative Commons license, users will need to obtain permission from the license holder to reproduce the material. To view a copy of this license, visit http://creativecommons.org/licenses/by/4.0/ 